\title{
Estudios naturalísticos versus estudios controlados en las recientes investigaciones sobre el tratamiento de la dependencia del alcohol
}

\author{
Jonathan Chick \\ Royal Edinburgh Hospital \\ Enviar correspondencia a:
}

Jonathan Chick. Departamento de Psiquiatría de la Universidad de Edimburgo. Royal Edinburgh Hospital. Edimburgo EH10 5 DX. Reino Unido.

\begin{abstract}
Resumen
Existen ventajas y desventajas en los estudios naturalísticos frente a los estudios controlados. Este documento examina alguna de las investigaciones recientes que pueden ayudar a aclarar el tema, partiendo de estudios que muestran los efectos si se reduce la duración en los tratamientos de los pacientes internos, estudios sobre el método "Doce pasos", procesos farmacoterapéuticos y Proyecto MATCH.

Es la propuesta del método "Doce pasos" la que ha conseguido un mayor apoyo, siendo sus efectos normalmente medicados a través de la asistencia de A.A. La conformidad con el tratamiento es un factor crítico en algunos estudios.
\end{abstract}

Palabras clave: evaluación, tratamiento alcoholismo, estudios controlados, estudios naturalísticos

\begin{abstract}
There advantages and disadvantages to the naturalistic versus the controlled study. This paper reviews some recent research to illustrate this, from studies showing effects of reducing length of in-patient treatment, studies of the Twelve-step method, pharmacotherapy trials, and Project MATCH. The Twelvestep approach has recently gained support, the effects usually medicated through A.A. attendance. Compliance with treatment is a critical factor in some studies.
\end{abstract}

Key words: evaluation, alcoholism treatment, controlled studies, naturalistic studies

Otra de las reservas con respecto a estas pruebas consiste en que, en un estudio aleatorio controlado, los pacientes están de acuerdo en ser asignados a uno de los tratamientos alternativos. Sin embargo, en el "mundo real" son los pacientes los que eligen qué tipo de tratamiento quieren recibir. Y los criterios que les empujan a una determinada elección pueden ser desconocidos para nosotros. A veces nos lo demuestran con la ayuda de sus pies: se van y nunca más vuelven a la siguiente cita, pues creen que el enfoque que hemos dado a su caso no es el más apropiado para el momento que atraviesan.

Pueden aparecer resultados interesantes cuando se permite a los pacientes que forman parte de un estudio controlado aleatorio pedir un tratamiento específico adicional. Besson y sus colaboradores, (1998) efectuaron un estudio aleatorio controlado de acamprosato versus placebo en alcohólicos suizos recientemente desintoxicados. En once de los doce estudios aleatorios controlados de acamprosato versus placebo realizados a lo largo de un año se lograron 
mejores resultados entre los pacientes que se hallaban en el grupo del acamprosato. A los pacientes que solicitaron que se añadiera disulfiram a su medicación durante el estudio, este fármaco les resultó especialmente beneficioso si estaban dentro del grupo del acamprosato. Resulta tentador sugerir que hay una interacción positiva entre la terapia con acamprosato y el uso de una medicación disuasiva: el disulfiram se relaciona con el ansia de beber a un nivel consciente, mientras el acamprosato ayuda a reducir un comportamiento condicionado de búsqueda de alcohol sin tener conciencia de ello. Lamentablemente, al no existir una distribución aleatoria de disulfiram, esto no puede ser más que una hipótesis cara a futuras pruebas; los pacientes que solicitan disulfiram pueden tener un mejor pronóstico porque se sienten más motivados de cara a la abstinencia que los pacientes que no la piden. Se podría argumentar lo contrario, esto es, que hay pacientes con una dependencia del alcohol más severa, y por ello, con más probabilidades de recaer.

Existen dos estudios recientes que comparan programas de mayor o menor duración en el ingreso de los pacientes con problemas de alcoholismo. Trent (1998) no encontró ninguna evidencia de peores resultados cuando, recientemente, la Marina de los E.E.U.U. redujo la duración de su programa para el tratamiento del alcoholismo con pacientes internos de seis a cuatro semanas. Una clínica inglesa privada mantuvo una estrecha vigilancia sobre 212 pacientes antes y durante un año después de seguir un tratamiento en el que se había reducido el programa de cinco semanas de ingreso hospitalario a solamente dos semanas como internos y con pacientes de día y externos asistidos, una alternativa mucho más barata. Se facilitaron repetidas citas para el seguimiento de ambos programas durante un año. Los resultados no fueron peores, basándose en los informes del paciente, de los familiares y en la mayoría de los casos de análisis de sangre: el 55\% no tenían problemas un año después de haber seguido cualquiera de los dos regímenes (Long y colaboradores, 1998). Algunas de las características de los pacientes fueron examinadas. Sin embargo, nos quedamos con la duda de que pudieran existir otras que los investigadores no hubieran controlado y que resultaran diferentes al acortar los programas. Los resultados de estos recientes estudios están en línea con las conclusiones de estudios controlados previos (pero para muchos médicos con experiencia, ello no es una razón para clausurar todas las camas dedicadas al tratamiento de pacientes alcohólicos -Schuckit, 1998).

Ninguno de estos estudios fue lo suficientemente amplio como para llevar a cabo múltiples y variados análisis que permitieran controlar las diferencias en las características de los pacientes. Un estudio en el "mundo real" de trece meses de duración (Ouimette y colaboradores, 1997) del VA (Veterans Affairs) manejó múltiples y variados análisis para ajustar los datos con arreglo a ciertas características previsibles en los pacientes. Compararon el resultado de pacientes tratados en clínicas siguiendo el programa "Doce pasos", cotejándolo con muestras de clínicas que trabajaban con tratamientos cognitivo-conductistas. Encontraron pocas mediciones que mostraran diferencias entre los dos tipos clínicos, aunque en los tratados en clínicas con métodos "Doce pasos" había una probable mayor abstinencia en el futuro como respuesta. Hasta el momento, los tratamientos "Doce pasos" no han sido examinados frente a programas de terapia cognitiva en estudios aleatorios controlados, y parece con este estudio que pueden ser igualmente eficaces, o quizá incluso más. Pero a pesar de los múltiples análisis, nos quedamos con la duda de si realmente ello sirve para defender los tratamientos "Doce pasos", pues nunca podremos saber si había algunas características del paciente sin evaluar o si existían diferencias en el modelo de reclutamiento para el tratamiento que hubieran podido influir en el resultado. Análisis múltiples y variados en un muestreo amplio permiten ajustes de muchas variables dudosas, pero jno se pueden ajustar variables que no han sido evaluadas!

EI Proyecto MATCH también reveló un resultado que surgió de un modo natural: que los pacientes que recibían tratamiento como internos mejoraban más que los que lo recibían solamente como externos (Grupo de investigación del Proyecto MATCH, 1997, 1998; Addiction, 1999).

El Proyecto MATCH no distribuía aleatoriamente a los pacientes en internos frente a externos. Existían dos ramas en el estudio, con reclutamiento de muestras de internos (el grupo "aftercare") y de pacientes que acudían a clínicas y que no habían asistido a ningún estudio previo como internos. El resultado fue que, en términos de consecución de la abstinencia, los pacientes de la rama "aftercare" lograban una mejora de un $15 \%$ con respecto a la rama de los externos. Sin embargo, es evidente que los pacientes de ambas ramas se podían haber seleccionado a sí mismos de un modo que no conocemos. Un efecto dudoso de este tipo podría ser que, por definición, los pacientes "aftercare" habían aceptado el tratamiento a lo largo de varias semanas y pacientes menos motivados podrían no haber superado la fase de internos. Asimismo, los primeros habían tomado su problema en serio hasta el punto de querer aceptar como primera opción el tratamiento como internos. Podrían haber estado en otro punto en la "historia natural" de su alcoholismo. Sin embargo, el resultado ha suscitado la cuestión de que algunos alcohólicos necesitan más de doce semanas de tratamiento externo, acudiendo a la consulta una vez por semana. Había una distribución aleatoria en cada rama del estudio ("aftercare" y pacientes externos), con ayuda del método 
“Doce pasos", terapia de comportamiento cognitivo o terapia de intensificación motivacional. Y de ese modo, a pesar de que el propósito principal era equiparar los efectos para determinar si algunos pacientes mejoraban con ciertos tratamientos, también se pueden efectuar comparaciones entre los resultados que se presentan en cada tipo de tratamiento.

\section{APOYO DEL PROYECTO MATCH AL MÉTODO DE} A.A.

"La propuesta Doce pasos", dando a conocer a los pacientes que el alcoholismo es una enfermedad y animando a los mismos a acudir a A.A., fue valorada por el Proyecto MATCH más positivamente de lo que había sido valorada en otras investigaciones. Como en el estudio naturalista VA, los datos mostraron que los efectos beneficiosos de este tipo de tratamiento se hallan apoyados por A.A. a través de una serie de reuniones posteriores. Los pacientes del Proyecto MATCH habrían sido consultados para su distribución en cada uno de los tres tipos de tratamiento, CBE, MET oTSF, e incluso muchos de ellos podrían haber tenido un conocimiento previo del la propuesta "Doce pasos," por la sencilla razón de que es muy conocida en EEUU, y es el primer lugar al que los pacientes suelen acudir, antes de acercarse a una organización donde se pierde el anonimato y que puede costar dinero. Ello puede dar lugar a ciertas preferencias: pacientes descontentos con el planteamiento de A.A se podrían haber excluido selectivamente del proyecto $\mathrm{MATCH}$, reduciendo de ese modo la generalización de los resultados. Sin embargo, el efecto comparativo, dentro de la muestra seleccionada, de que pacientes que habían vuelto a un entorno social caracterizado por un consumo elevado de alcohol mejoraban más con el tratamiento TSF que con otros, se evidencia como un resultado convincente (Longabough y colaboradores, 1998).

Antes de afirmar con seguridad de qué forma incide un tratamiento en los resultados obtenidos, debemos verificar si los pacientes realmente llevaron a cabo dicho tratamiento. Ni siquiera los pacientes especialmente seleccionados para el Proyecto MATCH asistieron a todas las sesiones de su tratamiento (a los pacientes no se les pagaba por asistir a las mismas, ja pesar de que sí les pagaban por acudir a las entrevistas!). Algunos han llegado a la conclusión de que el resultado de la muestra completa de MET (que comprendía tan sólo cuatro sesiones) asociada a resultados tan buenos como los de TSF y CBT (que comprendían doce sesiones), significa que las intervenciones breves podrían ser en términos generales tan efectivas como otras intervenciones de carácter más intensivo. Sin embargo, el número de citas atendidas fue de 3.2 en MET, 8.2 en CBT, 7.4 en TSF, más entrevistas personales a cada paciente en torno a doce horas durante el primer año. Por ello, la diferencia real entre los grupos en cuanto a intensidad de contacto no resultaba tan grande: los pacientes de TSF sólo tenían un $20 \%$ más que los de MET. Y éste no es más que un factor que puede haber oscurecido los resultados del Proyecto MATCH.

La conformidad se evidencia como un factor importante en recientes investigaciones con naltrexone. Nuestro estudio aleatorio controlado de naltrexone versus placebo sólo resultó más beneficioso en los resultados derivados de las muestras sanguíneas, serum gamma glutamyl transferasa (CGT) (Chick y colaboradores). Esto puede haber sido así porque el análisis GCT sólo tenía datos de pacientes que habían asistido a las citas y habían entregado muestras de sangre, es decir, que estaban de acuerdo con el tratamiento. En el subgrupo de los que se hallaban conformes con éste, predefinidos como aquéllos que asistieron a todas las citas y tomaron un $80 \%$ de los comprimidos recomendados, se advirtió la ventaja del naltrexone. Volpicelli y colaboradores (1997) comprobaron que sólo existía una ventaja estadísticamente significativa con respecto al naltrexone en aquellos pacientes que se mostraban conformes con el tratamiento. En pocas palabras, no podemos evaluar un tratamiento apoyándonos en aquellos pacientes que no lo siguen.

\section{CONCLUSIONES}

1. Los estudios controlados deben evitar criterios de exclusión excesivamente rigurosos en la búsqueda de su validez interna; como ya hemos indicado, sólo el 39\% de los pacientes investigados en el Proyecto Match formaron parte del estudio. Nuestro progreso puede ser favorecido por estudios naturalistas del modelo "antes" y "después", y con muestras suficientemente amplias para ajustarse a variables predecibles.

2. A la hora de evaluar un tratamiento, la conformidad debe ser potenciada y medida.

3. Pacientes internos de larga duración carecen aún de apoyo científico.

4. El enfoque de A.A. ha recibido el apoyo de distintos estudios científicos en los dos últimos años. A.A. parece resultar sumamente beneficioso para aquellos pacientes que no padecen síntomas psiquiátricos, y que viven en lugares donde el alcohol es socialmente aceptado y fácilmente asequible, por lo que existe una cierta presión hacia la bebida.

\section{BIBLIOGRAFÍA}

ADDICTION (1999) Commentaries on Project MATCH : matching alcohol treatments to client heterogeneity Addiction, 94, 31-69. 
Besson J, Aeby F, Kasas A, Lehert P and Potgieter A. (1998) Combined efficacy of acamprosate and disulfiram in the treatment of alcoholism : a controlled study Alcoholism: Clinical and Experimental Research 22, S73579.

Chick J, Drummond C, Moncrieff J. et al (en prensa) Naltrexone in the treatment of alcoholism British Journal of Psychiatry.

Long CG, Williams M, Hollín CR. (1998) Treating alcohol problems: a study of programme effectiveness and cost of effectiveness according to length and delivery of treatment. Addiction 93, 561-571.

Longabough R, Wirtz PW, Zweben A and Stout RL. (1998) Network support for drinking, Alcoholics Anonymous and long-term matching effects. Addiction 93, 13131334.

Ouimette PC, Finney J, Moos RH (1997) Twelve step and cognitive behavioural treatment for substance abuse : a comparison of treatment effectiveness. Journal of Consulting and Clinical Psychology 65, 230-240.

Project MATCH Research Group (1997) Matching alcoholism treatments to client group heterogeneity: Project MATCH post-treatment drinking outcomes. Journal of Studies on Alcohol, 58, 7-29.

Project MATCH Research Group (1997) Project MATCH secondary a priori hypotheses. Addiction 92, 16711698.

Schuckit M (1998) Penny-wise, Ton-foolish? The recent movement to abolish inpatient alcohol and drug treatment. Journal of Studies on Alcohol 59, 5-6.

Trent LK (1998) Evaluation of a four-versus six-week length of stay in the Navy's alcohol treatment program. Journal of Studies on Alcohol 59, 270-279.

Volpicelli JR, Rhines KC, Rhines JS, Volpicelli LA, Alterman Al, O’Brien CP. (1997) Naltrexone and Alcohol Dependence. Archives of General Psychiatry, 54, 737-42. 